\title{
Chromosomal Evolution and Apomixis in the Cruciferous Tribe Boechereae
}

\author{
Terezie Mandáková1, Petra Hloušková1, Michael D. Windham², Thomas Mitchell-Olds², \\ Kaylynn Ashby ${ }^{3}$, Bo Price ${ }^{3}$, John Carman ${ }^{3}$ and Martin A. Lysak ${ }^{1 *}$ \\ ${ }^{1}$ CEITEC, Masaryk University, Brno, Czechia, ${ }^{2}$ Department of Biology, Duke University, Durham, NC, United States, ${ }^{3}$ Plants, \\ Soils, and Climate Department, Utah State University, Logan, UT, United States
}

\section{OPEN ACCESS}

Edited by: Steven Dodsworth, University of Bedfordshire, United Kingdom

Reviewed by: Ana Paula Moraes, Universidade Federal do $A B C$, Brazil Aretuza Sousa Dos Santos, Ludwig Maximilian University

of Munich, Germany

${ }^{*}$ Correspondence: Martin A. Lysak martin.lysak@ceitec.muni.cz; lysak@sci.muni.cz

Specialty section: This article was submitted to Plant Systematics and Evolution, a section of the journal Frontiers in Plant Science

Received: 13 February 2020 Accepted: 06 April 2020

Published: 28 May 2020

Citation:

Mandáková T, Hloušková $P$ Windham MD, Mitchell-Olds T, Ashby K, Price B, Carman J and Lysak MA (2020) Chromosomal Evolution and Apomixis in the Cruciferous Tribe Boechereae.

Front. Plant Sci. 11:514 doi: 10.3389/fpls.2020.00514
The mustard family (Brassicaceae) comprises several dozen monophyletic clades usually ranked as tribes. The tribe Boechereae plays a prominent role in plant research due to the incidence of apomixis and its close relationship to Arabidopsis. This tribe, largely confined to western North America, harbors nine genera and c. 130 species, with $>90 \%$ of species belonging to the genus Boechera. Hundreds of apomictic diploid and triploid Boechera hybrids have spurred interest in this genus, but the remaining Boechereae genomes remain virtually unstudied. Here we report on comparative genome structure of six genera (Borodinia, Cusickiella, Phoenicaulis, Polyctenium, Nevada, and Sandbergia) and three Boechera species as revealed by comparative chromosome painting (CCP). All analyzed taxa shared the same seven-chromosome genome structure. Comparisons with the sister Halimolobeae tribe $(n=8)$ showed that the ancestral Boechereae genome $(n=7)$ was derived from an older $n=8$ genome by descending dysploidy followed by the divergence of extant Boechereae taxa. As tribal divergence post-dated the origin of four tribe-specific chromosomes, it is proposed that these chromosomal rearrangements were a key evolutionary innovation underlaying the origin and diversification of the Boechereae in North America. Although most Boechereae genera exhibit genomic conservatism, intra-tribal cladogenesis has occasionally been accompanied by chromosomal rearrangements (particularly inversions). Recently, apomixis was reported in the Boechereae genera Borodinia and Phoenicaulis. Here, we report sexual reproduction in diploid Nevada, diploid Sandbergia, and tetraploid Cusickiella and aposporous apomixis in tetraploids of Polyctenium and Sandbergia. In sum, apomixis is now known to occur in five of the nine Boechereae genera.

Keywords: apomixis, apospory, autopolyploidy, Cruciferae, descending dysploidy, karyotype evolution, North America, speciation

\section{INTRODUCTION}

Geographically well-defined clades provide ideal study systems for understanding the role of whole-genome duplications (WGDs, polyploidy) and chromosomal rearrangements in speciation and diversification. Frequently, a group of species confined to an island, mountain range, or (sub)continent is assumed to have originated in this region, perhaps following 
an earlier dispersal event from another part of the world (e.g., Linder and Barker, 2014; Givnish et al., 2016). With the advent of molecular phylogenetics, in many cases, the inferred monophyly of a group has confirmed its geographical determinant and helped to elucidate its origin as well as directionality of later migrations and dispersals (e.g., Cowie and Holland, 2008; Ogutcen and Vamosi, 2016; Huang et al., 2018; Carter et al., 2019; Kim et al., 2019). A geographically restricted clade might be supported by different synapomorphies, such as morphological traits, specific metabolic pathways, pollination syndromes, or a shared WGD, some falling in the category of rare genomic changes (RGCs, Rokas and Holland, 2000). Structural chromosomal changes may underlie incipient reproductive isolation inducing species splits and the evolution of separate gene pools, i.e., cladogenesis (Faria and Navarro, 2010). Dysploidal (i.e., chromosome number changes caused by fusions and fissions) as well as non-dysploidal (i.e., deletions, duplications, inversions, and translocations) chromosomal rearrangements can modify recombination frequency, gene expression, the duration of cellular processes (replication, mitosis, and meiosis), and the degree of infertility of heterozygous hybrids. Thus, some chromosomal rearrangements may precipitate lineage splitting yet occur within a monophyletic clade (Freyman and Höhna, 2017).

The economically important mustard family (3977 species in 351 genera, BrassiBase ${ }^{1}$, accessed on February 1,2020) radiated into four (Franzke et al., 2011) to six (Huang et al., 2016) lineages or super-tribes $\sim 23$ million years ago (Hohmann et al., 2015). These lineages have been divided into 52 monophyletic tribes (BrassiBase) ranging in size from the monospecific Shehbazieae (German and Friesen, 2014) to the Arabideae, which harbors more than 390 species (Jordon-Thaden et al., 2013; Karl and Koch, 2013). Many crucifer tribes do not differ in their basal or ancestral chromosome numbers. For example, tribes of lineage II/B and lineage III/E have the same number of ancestrally shared linkage groups and the same dysploid chromosomal rearrangements (Mandáková and Lysak, 2008; Mandáková et al., 2017a). By contrast, tribes of lineage I/A, such as Boechereae $(x=7)$, Descurainieae $(x=7)$, Erysimeae (mostly $x=7)$, Turritideae $(x=6)$, and Yinshanieae $(x=6$ and 7) (Warwick and Al-Shehbaz, 2006; BrassiBase), appear to represent tribes that originated after independent reductions of the ancestral chromosome number $(n=8)$ to $n=7$ and $n=6$. None of the diploid Brassicaceae tribes with a clade-specific descending dysploidy have been investigated genomically, so it remains unclear whether intra-tribal diversification (i.e., speciation and origin of new genera) has involved non-dysploidal chromosomal rearrangements.

Here we focus on the tribe Boechereae which harbors c. 130 species. The vast majority of Boechereae taxa occurs only in North America, with one of these extending to Greenland and three species being endemic to the Russian Far East (Alexander et al., 2013; Doudkin and Volkova, 2013). Molecular studies (Beilstein et al., 2010; Nikolov et al., 2019) using various chloroplast and nuclear gene markers support the Boechereae (with a shared chromosome base number of $x=7$ ) as a

${ }^{1}$ https://brassibase.cos.uni-heidelberg.de/ monophyletic clade sister to the New World tribe Halimolobeae $(x=8 ; 39$ species in five genera, Al-Shehbaz, 2012). Alexander et al. (2013) recognized nine genera of Boechereae, seven of which (Anelsonia J. F. Macbride and Payson, Cusickiella Rollins, Nevada N. H. Holmgren, Phoenicaulis Nuttall, Polyctenium Greene, Sandbergia Greene, and Yosemitea P. J. Alexander and Windham) are mono- or bispecific, and, except for Sandbergia whitedii (Piper) Greene, restricted to the western United States. Boechera Á. Löve and D. Löve is by far the most diverse genus of the tribe, largely confined to the western part of the North American continent (Alexander et al., 2013). One group of eight species often assigned to Boechera was transferred to the genus Borodinia N. Busch by Alexander et al. (2013). This species group has the most discrete geographic range, apparently restricted to eastern North America and the Russian Far East [Borodinia macrophylla (Turcz.) O. E. Schulz]. Despite their largely allopatric distributions, Boechera and Borodinia species have hybridized in nature to produce one widespread sexual tetraploid and a series of apomictic triploids and tetraploids that erase any morphological distinctions between the two genera (Windham et al., 2014). When subsumed within Boechera, these lineages are informally designated the "western" and "eastern" clades, respectively.

The species now assigned to Boechera $(x=7)$ were originally included in Arabis L. (tribe Arabideae; $x=8$ ), but a series of molecular analyses (Koch et al., 2000; Beilstein et al., 2010; Nikolov et al., 2019) has shown that these genera belong to different major lineages of Brassicaceae. Boechera is phylogenetically closely related to the model genus Arabidopsis Heynh. (Figure 1A) and is best known for its classic agamic complex consisting of numerous, morphologically diverse, facultative, and obligate gametophytic apomicts. These are generally of hybrid origin, arising from a diverse array of sexual diploids with more restricted habitats. The genus is named for Danish botanist Tyge Böcher, who first documented apomixis in Boechera holboellii (Hornem.) Á. Löve and D. Löve (Böcher, 1951). The relatively close relationship of these species to Arabidopsis, combined with its diversity of ploidies (Alexander et al., 2015) and apomixis types, at both the diploid and polyploid levels (Carman et al., 2019), have made the genus a major focus for apomixis research (e.g., Naumova et al., 2001; Schranz et al., 2005; Lee et al., 2017; Kliver et al., 2018; Rojek et al., 2018; Brukhin et al., 2019). Until recently, apomixis within Boechereae was thought to be confined to the large genus Boechera. However, Mandáková et al. (2020) documented the occurrence of apomixis at the diploid, triploid, and tetraploid levels in one of the smaller genera of Boechereae (Phoenicaulis), raising the possibility that apomixis might also occur in other Boechereae genera.

In flowering plants, apomixis can be defined as asexual seed formation where clonal embryos originate either from unreduced eggs produced in unreduced female gametophytes (gametophytic apomixis) or from somatic cells of the ovule wall without an intervening unreduced gametophyte generation (sporophytic apomixis). In sporophytic apomixis, a reduced gametophyte forms, which supports the clonal embryo while it develops. The reduced gametophyte may or may not contain a sexually derived embryo (Asker and Jerling, 1992; Hand and Koltunow, 2014). Gametophytic apomixis is a prominent mode of reproduction 
A

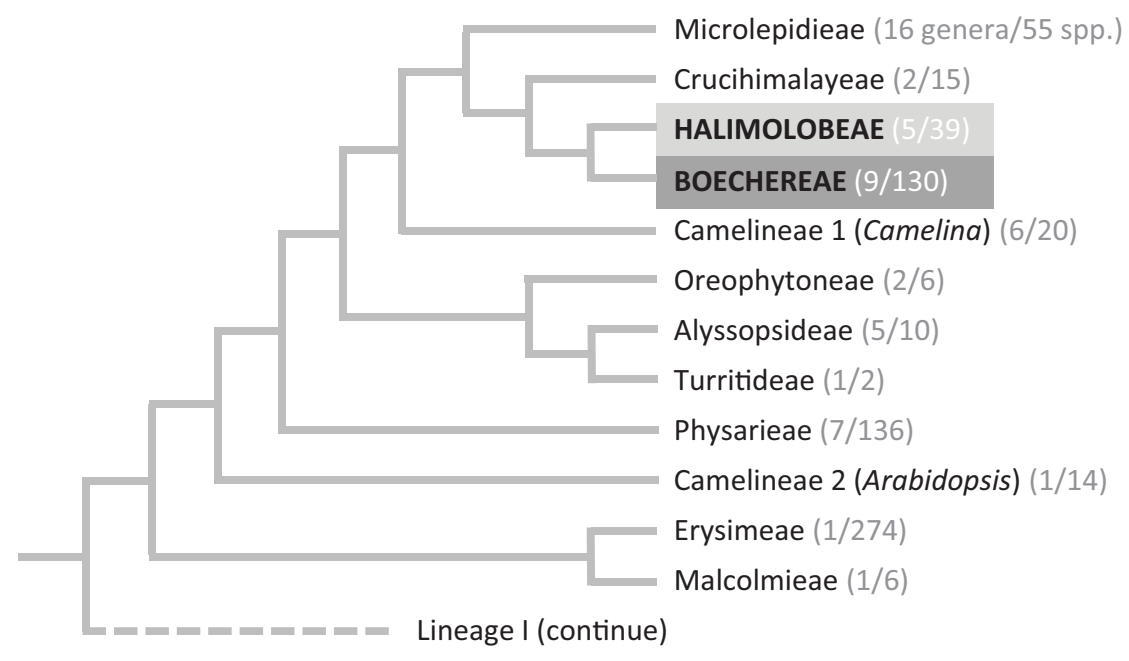

B

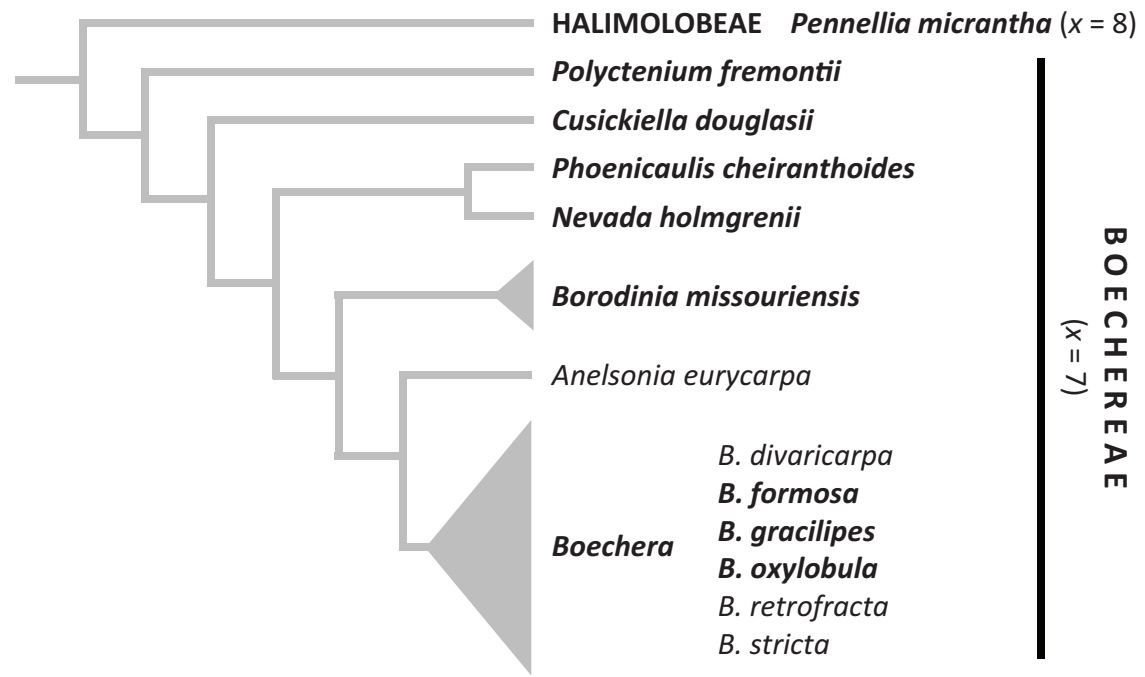

FIGURE 1 | (A) Phylogenetic position of the Boechereae in the Brassicaceae based on Nikolov et al. (2019). In parentheses, the number of genera and species is given based on data from BrassiBase (https://brassibase.cos.uni-heidelberg.de/). (B) Generic relationships within the Boechereae based on Beilstein et al. (2010) (Sandbergia and Yosemitea not shown).

in Boechera (Böcher, 1951; Roy, 1995; Naumova et al., 2001; Schranz et al., 2005), where it has greatly increased the diversity of genotypes and phenotypes by stabilizing the products of reticulate evolution (Beck et al., 2012; Carman et al., 2019). Three types of gametophytic apomixis occur in Boechera, and these are differentiated based on where the unreduced gametophyte forms: (i) if from a megasporocyte [megaspore mother cell (MMC)], it is referred to as Antennaria type diplospory, (ii) if from an apomeiotic dyad member of a first division meiotic restitution event, it is referred to as Taraxacum type diplospory, and (iii) if from a nucellar or parietal cell, it is referred to as Hieracium type apospory (Carman et al., 2019). The Antennaria type appears to be an oddity in Boechera that has been observed only rarely in plants that otherwise reproduce by Taraxacum type diplospory. In contrast, Taraxacum type diplospory and apospory are more commonly encountered in natural populations of Boechera than is sexual reproduction (Carman et al., 2019).

In the light of the fragmentary knowledge of genome evolution and reproductive modes in the Boechereae, we embarked on comparative cytogenetic and embryological analysis of several taxa representing the tribal diversity. We followed several aims: (1) To expand cytogenomic sampling of the species and genera of Boechereae to provide a more complete understanding of chromosomal evolution in the tribe, (2) to determine whether diversification within the tribe was accompanied by clade-specific chromosomal rearrangement, (3) to test whether an ancestral 
$n=7$ genome inferred for Boechera and Phoenicaulis (Mandáková et al., 2015b, 2020) provides an accurate reconstruction of the ancestral genome of the tribe as a whole, and (4) to gain insights into the reproductive modes of previously unsampled Boechereae genera by conducting embryological analyses on most of the species analyzed cytogenomically.

\section{MATERIALS AND METHODS}

\section{Species Analyzed}

Ten Boechereae species were selected to span the phylogenetic diversity of the tribe (Figure 1). These included Boechera formosa (Greene) Windham and Al-Shehbaz $(2 n=14)$, Boechera gracilipes (Greene) Dorn $(2 n=14)$, Boechera oxylobula (Greene) W. A. Weber $(2 n=14)$, Borodinia missouriensis (Greene) P. J. Alexander and Windham $(2 n=14)$, Cusickiella douglasii (A. Gray) Rollins $(2 n=28)$, Nevada holmgrenii (Rollins) N. H. Holmgren $(2 n=14)$, Phoenicaulis cheiranthoides $(2 n=14,21,28)$, Polyctenium fremontii (S. Watson) Greene $(2 n=28)$, Sandbergia perplexa (L. F. Henderson) Al-Shehbaz $(2 n=14)$, and S. whitedii $(2 n=21)$. As the tribe Halimolobeae was repeatedly retrieved as a sister clade to Boechereae (e.g., Beilstein et al., 2010; Couvreur et al., 2010; Alexander et al., 2013), Pennellia micrantha (A. Gray) Nieuwland $(2 n=16)$ was selected to represent the Halimolobeae clade outgroup. The origins of the analyzed populations are listed in Supplementary Table S1; multiple individuals were analyzed from each population.

The analyzed plants were either collected in the wild or grown in a growth chamber from seeds collected in the wild. Young inflorescences of the analyzed plants were collected and fixed in freshly prepared fixative (ethanol: acetic acid, 3: 1) overnight, transferred to $70 \%$ ethanol and stored at $-20^{\circ} \mathrm{C}$.

\section{Chromosome Preparation}

Chromosome spreads from fixed young flower buds containing immature anthers were prepared according to published protocols (Lysak and Mandáková, 2013; Mandáková and Lysak, 2016a). Chromosome preparations were treated with $100 \mu \mathrm{g} / \mathrm{ml}$ RNase in $2 \times$ sodium saline citrate (SSC; $20 \times$ SSC: 3 M sodium chloride, $300 \mathrm{mM}$ trisodium citrate, $\mathrm{pH} 7.0$ ) for $60 \mathrm{~min}$ and with $0.1 \mathrm{mg} / \mathrm{ml}$ pepsin in $0.01 \mathrm{M} \mathrm{HCl}$ at $37^{\circ} \mathrm{C}$ for $5 \mathrm{~min}$, then postfixed in $4 \%$ formaldehyde in distilled water, and dehydrated in an ethanol series (70, 90, and 100\%, 2 min each).

\section{DNA Probes}

The BAC clone T15P10 (AF167571) of Arabidopsis thaliana (L.) Heynh. bearing 35S rRNA gene repeats was used for in situ localization of nucleolar organizer regions (NORs), and the A. thaliana clone pCT4.2 (M65137), corresponding to a $500 \mathrm{bp}$ $5 \mathrm{~S}$ rDNA repeat, was used for localization of $5 \mathrm{~S}$ rDNA loci. For Comparative Chromosome Painting (CCP), 674 chromosomespecific BAC clones of $A$. thaliana (The Arabidopsis Information Resource, $\mathrm{TAIR}^{2}$ ) were used to establish contigs corresponding

${ }^{2}$ http://www.arabidopsis.org to the 22 genomic blocks (GBs) and eight chromosomes (AK1AK8) of the Ancestral Crucifer Karyotype (ACK; Lysak et al., 2016). See Supplementary Tables S2-S8 for the list of BAC clones used to identify the 22 GBs on chromosomes of the Boechereae species. To determine and characterize inversions and split GBs, some BAC contigs were split into smaller subcontigs and differentially labeled (e.g., Aa, Ab, Ca, Cb, see Supplementary Tables S3-S8). All DNA probes were labeled with homemade biotin-dUTP, digoxigenin-dUTP, or Cy3-dUTP by nick translation as described by Mandáková and Lysak (2016b).

\section{Comparative Chromosome Painting (CCP)}

DNA probes were pooled to follow the design of a given experiment, ethanol precipitated, dried, and dissolved in $20 \mu \mathrm{l}$ of $50 \%$ formamide and $10 \%$ dextran sulfate in $2 \times$ SSC. The $20 \mu \mathrm{l}$ of the dissolved probe were pipetted on a chromosomecontaining slide and immediately denatured on a hot plate at $80^{\circ} \mathrm{C}$ for $2 \mathrm{~min}$. Hybridization was carried out in a moist chamber at $37^{\circ} \mathrm{C}$ overnight. Post-hybridization washing was performed in $20 \%$ formamide in $2 \times$ SSC at $42^{\circ} \mathrm{C}$ three times (5 min each time). Hybridized probes were visualized either as the direct fluorescence of Cy3-dUTP or through fluorescently labeled antibodies against biotin-dUTP and digoxigenin-dUTP following Mandáková and Lysak (2016b). Chromosomes were counterstained with 4',6-diamidino2-phenylindole (DAPI, $2 \mu \mathrm{g} / \mathrm{ml}$ ) in Vectashield antifade. Fluorescence signals were analyzed and photographed using a Zeiss Axioimager epifluorescence microscope with a CoolCube camera (MetaSystems). Images were acquired separately for all four fluorochromes using appropriate excitation and emission filters (AHF Analysentechnik). The four monochromatic images were pseudocoloured, merged, and cropped using Photoshop CS (Adobe Systems) and ImageJ (National Institutes of Health).

\section{In silico Sequence Analysis}

Boechera stricta (Graham) Al-Shehbaz (v1.2; Lee et al., 2017), Arabidopsis lyrata (L.) O'Kane and Al-Shehbaz (v2.1; Hu et al., 2011), and A. thaliana (TAIR 10) genome assemblies and annotations were downloaded from the Phytozome webpage ${ }^{3}$. Inter-genome collinearity was analyzed by SynOrths, identifying whether two homeologous genes are a conserved syntenic pair based on their sequence similarity and the support of homeologous flanking genes (Cheng et al., 2012).

\section{Embryological Analyses}

Clusters of pre-anthesis staged floral buds were fixed in 3:1 fixative for $48 \mathrm{~h}$ and stored in $70 \% \mathrm{EtOH}$. Ovaries were excised, cleared, measured, and mounted following Mandáková et al. (2020). An Olympus (Center Valley, PA, United States) BX53 microscope with differential interference contrast (DIC) optics and equipped with a DP74 digital camera with cellSens Dimension 1 software (Olympus) was used to investigate parietal cell, MMC, and female gametophyte origins.

\footnotetext{
${ }^{3}$ https://phytozome.jgi.doe.gov/pz/portal.html
} 


\section{RESULTS}

Based on the ACK, and on previously analyzed Boechereae species (Mandáková et al., 2015a, 2020), detailed comparative cytogenetic maps were constructed by CCP for each of the 10 Boechereae species and for $P$. micrantha (Figure 2 and Supplementary Tables S2-S8).

\section{The Outgroup Pennellia micrantha Genome Structurally Mirrors the ACK}

Comparative chromosome painting in $P$. micrantha $(2 n=16$, Halimolobeae) was successful in identifying all 22 conserved GBs making up the eight chromosomes (Hal1-Hal8, Figures 2, 3 and Supplementary Table S2). The ACK-like Pennellia genome further corroborrated the earlier assumption (Mandáková et al., 2015b, 2020) that the Most Recent Common Ancestor (MRCA) of Boechereae and Halimolobeae had eight chromosomes and structurally resembled the ancestral genome of crucifer lineage I.

\section{Overall Structural Stasis of Boechereae Genomes}

Comparative chromosome painting with painting probes designed according to the structure of the seven linkage groups in Boechera (Mandáková et al., 2015a) and Phoenicaulis (Mandáková et al., 2020), were effective in identifying all seven or 14 chromosome pairs among the 10 Boechereae species analyzed. All 10 genomes had a very similar organization (Figure 2), except for a few species-specific chromosomal rearrangements (see below). The overall structural genome similarity among different Boechereae genera allowed us to reconstruct the genome of the MRCA of Boechereae.

\section{Ancestral Boechereae Genome}

By comparing the 10 Boechereae genomes studied herein with those of three diploid Boechera taxa (Mandáková et al., 2015b), Phoenicaulis (Mandáková et al., 2020), and P. micrantha, we inferred the ancestral Boechereae genome with seven pairs of chromosomes (Boe1-Boe7, Figures 2, 3). Three of these pairs (Boe4, Boe6, and Boe7) retained their ancestral structure as in the ACK, or Halimolobeae, whereas four pairs (Boe1-Boe3 and Boe5) are specific to the Boechereae genomes (Figures 2-4 and Supplementary Table S3).

CCP chromosome painting analyses allowed us to reconstruct the origin of the four Boechereae-specific chromosomes. The origin of chromosomes Boel and Boe2 (Figure 4A) most likely included an initial $0.52-\mathrm{Mb}$ pericentric inversion on ancestral chromosome AK1 with breakpoints within GBs A [between BAC clone F13B4 (At1g13620) and T16N11 (At1g15410)] and C [between F8L10 (At1g53170) and F12M16 (At1g53160)]. The size of this inversion and other documented rearrangements were inferred from the physical length $(\mathrm{Mb})$ of $A$. thaliana BAC contigs spanning these chromosome regions. The inversion-bearing AK1 chromosome underwent a whole-arm translocation with paleochromosome AK2 resulting in chromosomes Boe1 (GBs Aa, $\mathrm{Ca}$, and $\mathrm{D}$ ) and Boe2 (Cb, Ab, B, and E). Chromosome Boe3 (F, $\mathrm{G}, \mathrm{W}$, and $\mathrm{X}$ ) originated by a whole-arm translocation between paleochromosomes AK3 and AK8 (Figure 4B). The second translocation chromosome (GBs $\mathrm{V}$ and $\mathrm{H}$ ) was involved in an end-to-end translocation with chromosome AK5, mediating the chromosome number reduction $(8 \rightarrow 7)$ in Boechereae. The collinearity of GBs K-L and $\mathrm{M}-\mathrm{N}$ and the absence of the original centromere suggest that the "chromosome fusion" was accompanied or followed by a removal of the AK5 paleocentromere (Figure 4B). Remnants of the AK5 paleocentromere, apparent as heterochromatic knobs and/or unpainted chromosome segments, were not observed in any of the analyzed species (Figures 3, 4B).

\section{Clade-Specific Chromosomal Rearrangements}

The ancestral Boechereae genome remained conserved in the diploid B. gracilipes (Figures 2, 3 and Supplementary Table S3) and the tetraploid $P$. fremontii (Figure $\mathbf{2}$ and Supplementary Table S3). Given that both Polyctenium subgenomes had identical chromosome structure and the pachytene chromosomes formed quadrivalents (Figure 5), the analyzed accession of $P$. fremontii was most likely of autotetraploid origin.

In the three cytotypes of $P$. cheiranthoides $(2 x, 3 x$, and $4 x$; Mandáková et al., 2020), diploid N. holmgrenii, diploid S. perplexa, and triploid S. whitedii, chromosome Boe4 was altered by an $8.24-\mathrm{Mb}$ paracentric inversion spanning the entire block J (whole long arm). The breakpoints were located in the pericentromeric and subtelomeric regions (Figures 2, 6 and Supplementary Tables S4, S5). The absence of subgenome differentiation in the analyzed triploid and tetraploid populations of Phoenicaulis and S. whitedii suggests intraspecific, autopolyploid origins for these polyploids. Additionally, $N$. holmgrenii exhibited a $2.55-\mathrm{Mb}$ whole-arm pericentric inversion on Boe5. In this population, the short arm (GB V) was inverted, rendering the acrocentric chromosome telocentric (Figures 2, 6 and Supplementary Table S5).

Chromosome Boe4 of tetraploid C. douglasii also displayed inversions (Figure 2 and Supplementary Table S6). A 2.06$\mathrm{Mb}$ pericentric inversion, spanning the whole short arm (GB I), converted the chromosome from acrocentric to telocentric. Boe4 was also modified by a $5.35-\mathrm{Mb}$ paracentric inversion [breakpoint between blocks I and J, and within block Jbetween T28M21 (At2g40090) and T3G21 (At2g40240)]. The Cusickiella population analyzed for this study was most likely of autotetraploid origin given that both subgenomes were structurally similar, including a reshuffling of Boe4 (data not shown). Finally, in B. formosa and B. oxylobula, Boe5 was altered by a $9.83-\mathrm{Mb}$ pericentric inversion with breakpoints within block $\mathrm{V}$ [between K14A3 (At5g47175) and MQL5 (At5g47150)] and K-L [between MQP15 (At3g30655) and MED5 (At3g30663)], converting the chromosome from acrocentric to metacentric (Figures 2, 6 and Supplementary Table S7).

Borodinia missouriensis had the most reshuffled genome encountered among the taxa analyzed (Figures 2, 6 and Supplementary Table S8). A whole-arm translocation between Boe1 (GBs Aa, Ca, and D) and Boe3 (F, G, W, and X) produced two B. missouriensis-specific translocation chromosomes. The 


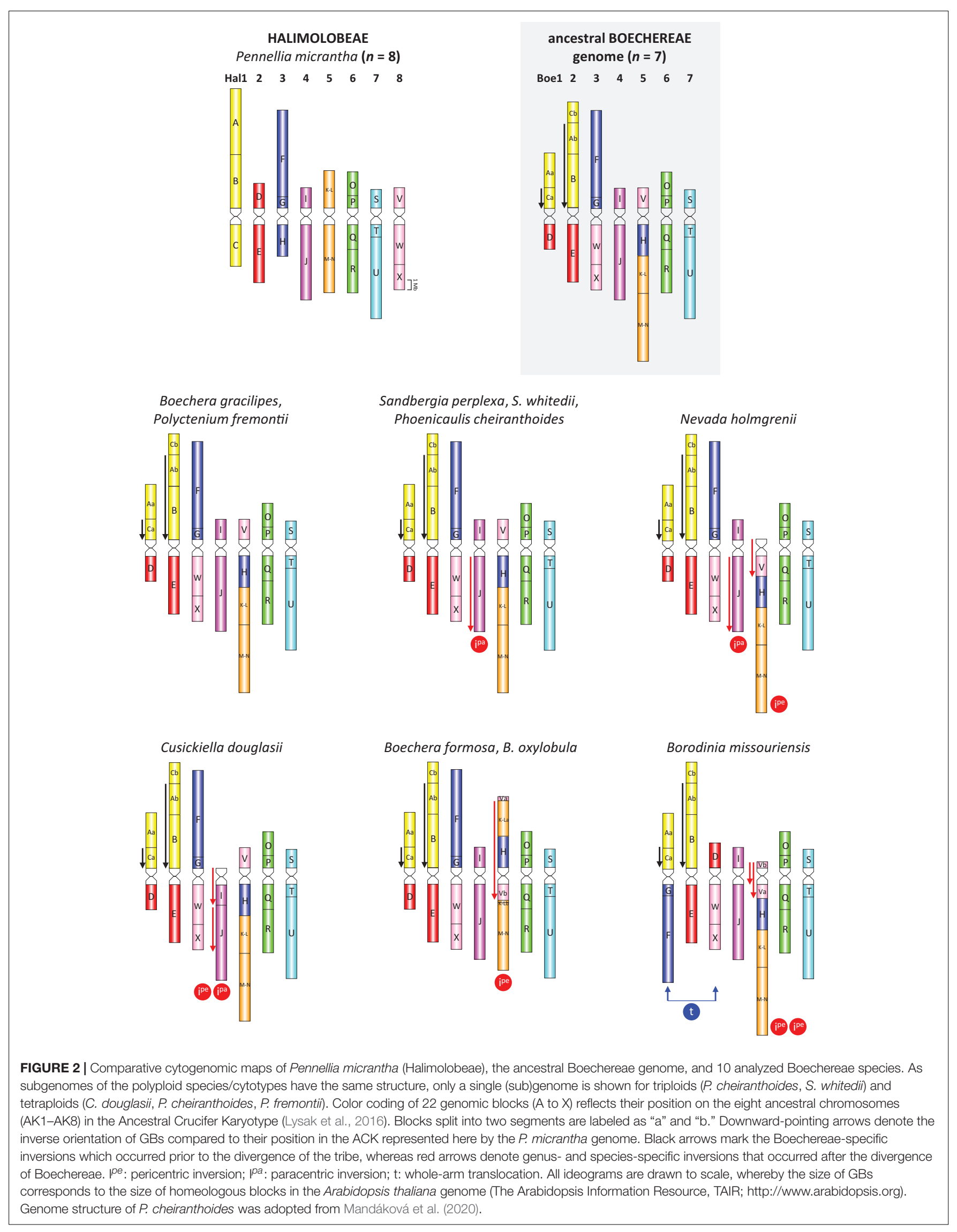




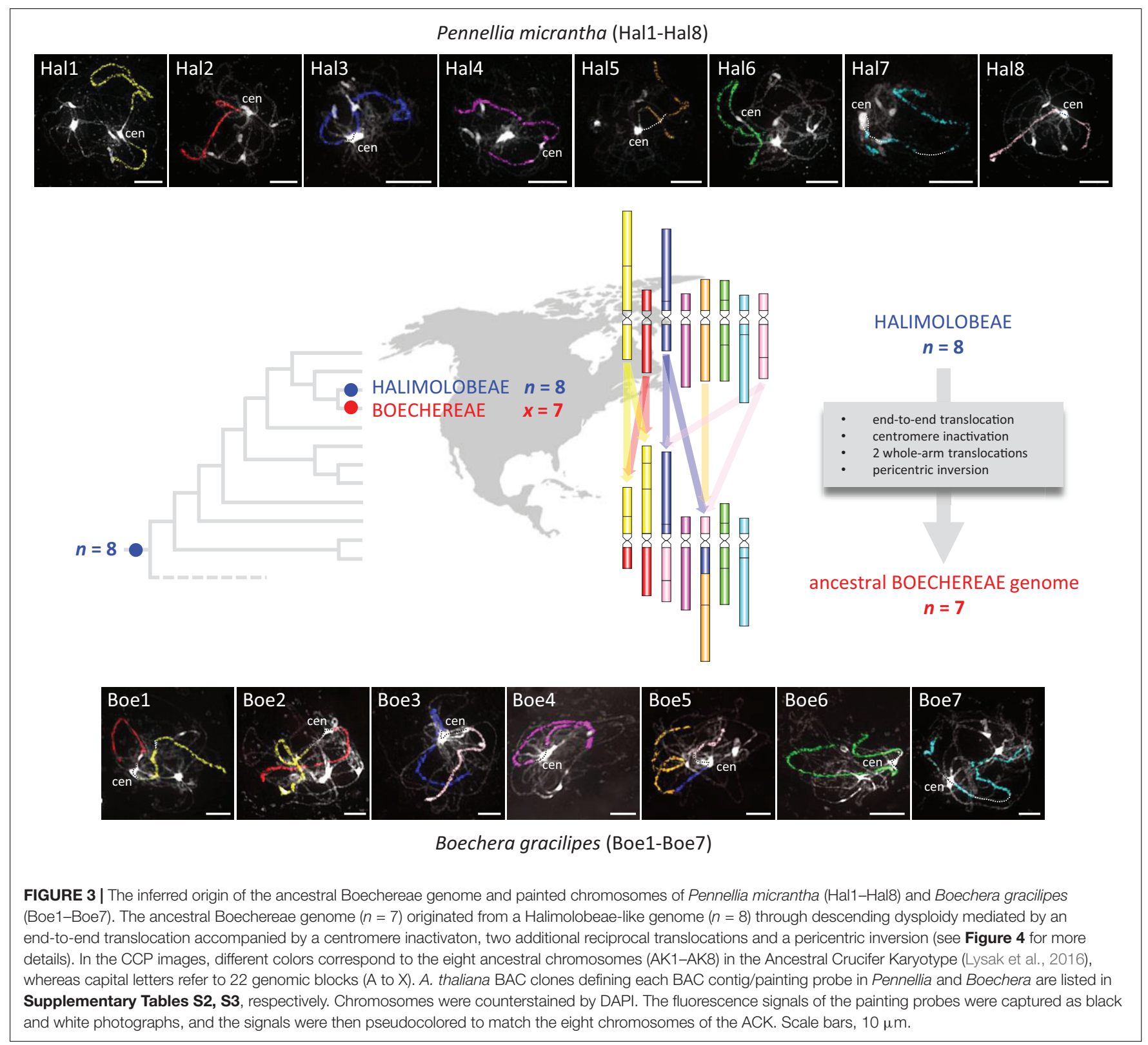

species also shared a $2.55-\mathrm{Mb}$ whole-arm pericentric inversion spanning block $\mathrm{V}$ of Boe 5 with $N$. holmgrenii. In B. missouriensis, this was followed by a small $0.57-\mathrm{Mb}$ pericentric inversion splitting block $\mathrm{V}$ into $\mathrm{Va}$ and $\mathrm{Vb}$ and placing the Boe5 centromere close to the chromosome terminus between BACs K24F5 (At5g43211) and MNL12 (At5g43190).

\section{Localization of rDNA Loci}

In $P$. micrantha, NORs (35S rDNA loci) were localized on the termini of five chromosomes (Hall, Hal3, Hal4, Hal6, and Hal7) and two 5S rDNA loci were adjacent to the pericentromeric heterochromatin of chromosomes Hal4 and Hal6 (Supplementary Figure S1). A single NOR and 5S rDNA locus were identified in eight Boechereae species analyzed; two NORs were found in Cusickiella and two 5S loci in Nevada
(Supplementary Figure S1). NORs were located terminally on the short chromosome arm of Boe4 (Boechera spp. and Cusickiella) or Boe5 (Borodinia, Cusickiella, Polyctenium, and Nevada). In Phoenicaulis and both Sandbergia species, NORs were located interstitially, close to the pericentromere of Boe6. This supports the close relationship between Phoenicaulis and Sandbergia. Interestingly, all telocentric chromosomes were NOR-bearing (Boe4 in Cusickiella and Boe5 in Borodinia and Nevada). 5S rDNA loci were found positioned interstitially, close to the pericentromere of Boe5 (Cusickiella, Nevada, and Phoenicaulis), Boe6 (Boechera spp. and Polyctenium), and Boe7 (Borodinia, Nevada, and both Sandbergia spp.).

In triploid (Phoenicaulis, $S$. whitedii) and tetraploid (Cusickiella, Phoenicaulis, Polyctenium) taxa/cytotypes, the position of rDNA gene loci at the same chromosomal positions 


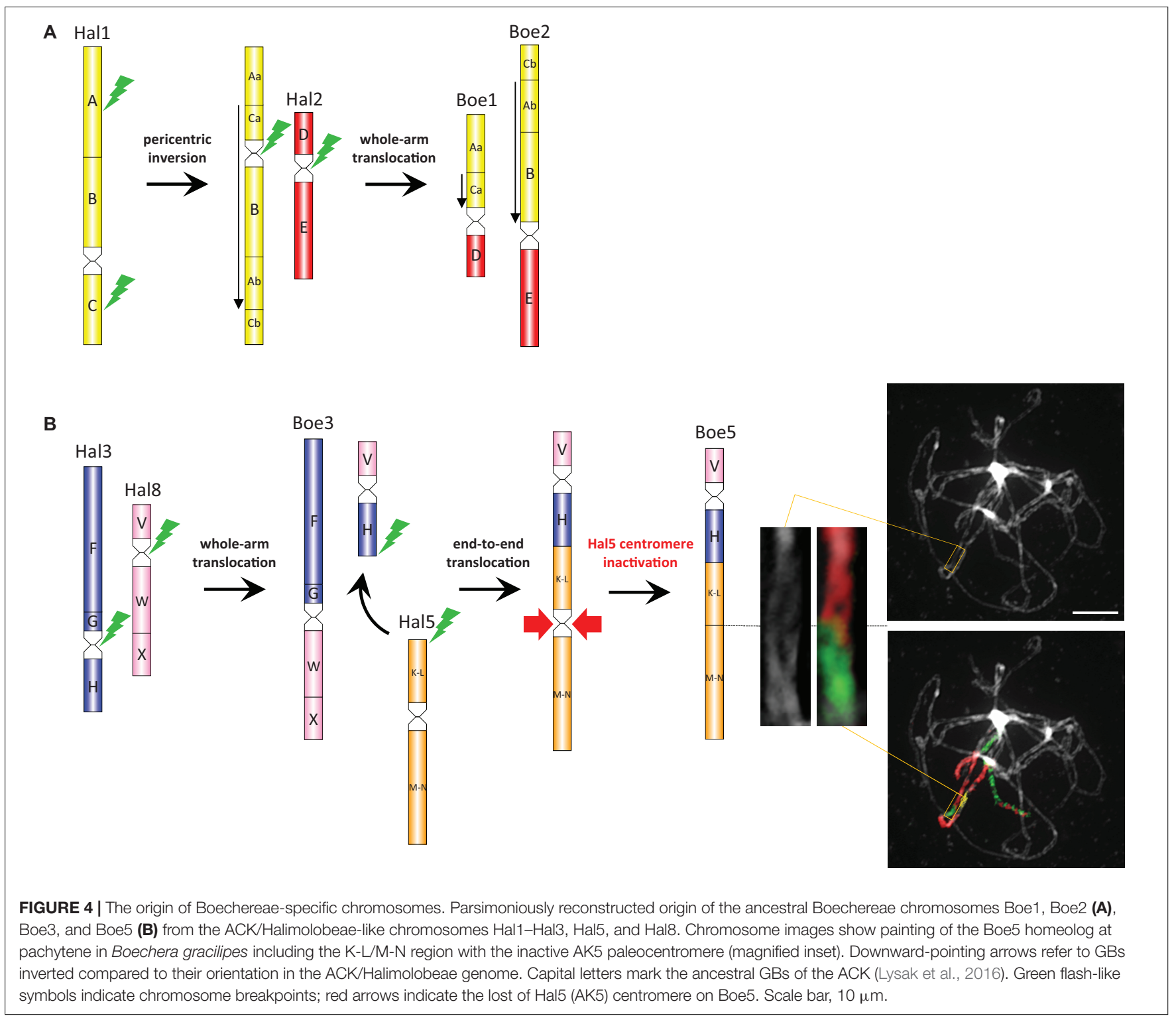

within three (triploid) or four (tetraploid) chromosome sets, further supported the purported autopolyploid origins of these genomes.

\section{The Inactive Centromere Between Genomic Blocks K-L and M-N in Boechera stricta}

The B. stricta genome contains an inactive centromere between GBs K-L and M-N on chromosome Boe5. To characterize this region at the sequence level, we compared scaffold 556 in the $B$. stricta assembly with orthologous genes on homeologous chromosome 3 and 5 in A. thaliana and A. lyrata, respectively (Figure 7A). The centromeric region is delimited by genes of the Peroxidase superfamily (loci At3g32980) and genes of the Transducin family (loci At3g33530). In B. stricta, the site of the eliminated (AK5) paleocentromere corresponds to a 13-kb region between orthologs Bostr.0556s0638 and Bostr.0556s0640. This region contains a single gene (Bostr.0556s0639), which is presumably paralogous to gene Bostr.13158s0074 (homology of $84.9 \%$ ), located in the distant part of block M-N on the same chromosome. In the Arabidopsis genomes, orthologs of Bostr.13158s0074 are located within M-N on chromosomes At3 (At3g60740; At3: 22,447,245-22,453,364) and Al5 (scaffold_503309.1; Al5: 20,028,781-20,034,751), respectively. Additionally, the A. lyrata genome possesses a paralog, Al_scaffold_0002_1021, located on Al2 in GB E (position 9,412,104-9,422,712). No remnants of tandem repeats were detected within the $13-\mathrm{kb}$ region (Figure 7B). Comparable distribution of transposable elements and their remnants was observed within the former centromeric region, in upstream and downstream $20 \mathrm{~kb}$ regions (Figure $7 \mathrm{C}$ ) and along the whole Boe5 pseudo-chromosome. The absence of repeat segment enrichment within the short $13-\mathrm{kb}$ region supports an almost complete 


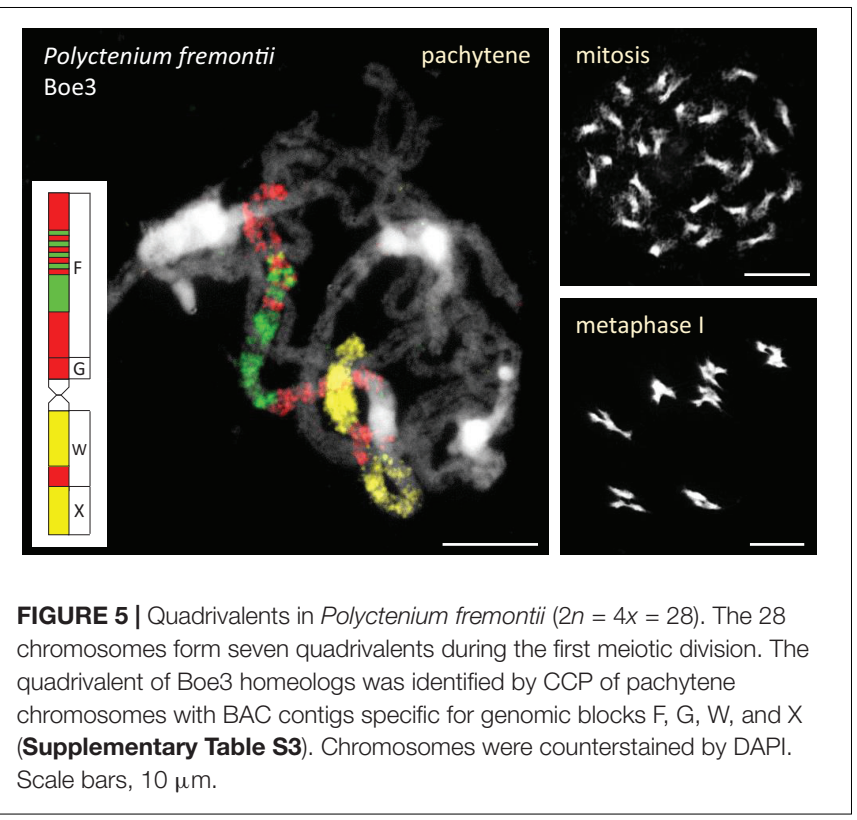

removal of the AK5 paleocentromere after the end-to-end "chromosome fusion" (Figure 4B).

\section{The Heterochromatic Het Chromosome Was Absent in the Analyzed Boechereae Species}

In apomictic Boechera species $(2 n=14)$ and Phoenicaulis cytotypes $(2 n=21$ and 28 , but not apomictic $2 n=14)$, one of the Boel homologs (a Het chromosome) displayed expanded regions of pericentromeric heterochromatin (Mandáková et al., 2015a, 2020). In all analyzed species of the present study, pericentromeric heterochromatin regions of the Boel homologs were comparable in size.

\section{High Frequency Apospory Occurs in Polyctenium and Sandbergia}

Six accessions (Supplementary Table S1), representing five species distributed across four genera, were embryologically analyzed (Figure 8). Tetraploid C. douglasii, diploid N. holmgrenii, and diploid S. perplexa, were sexual. Tetraploid P. fremontii (MDW 2055, ES 1078) and triploid S. whitedii were highly aposporous. In the $P$. fremontii population ES 1078, the dyad to tetrad ratio was high (Figure 8), which in Boechera would generally indicate diplospory (Carman et al., 2019). However, three of the four observed gametophytes were forming from nucellar cells (apospory), and the fourth was forming from the surviving megaspore of a sexual tetrad. While this sample size is too small to rule out diplospory, our observations indicated that apospory initiates early during ovule development with meiosis regularly terminating as early as the sexual dyad to early tetrad stages. Such termination may have inflated the dyad to tetrad ratio (Figure 8).

Parietal cell frequencies were $\geq 70 \%$ for five of the six taxa studied (Figure 8). These frequencies are similar to those observed in Phoenicaulis (Mandáková et al., 2020), but they are much higher than those generally observed in Boechera (<50\%) (Naumova et al., 2001; Carman et al., 2019). The ES 1078 P. fremontii sample was too small to determine this frequency. Parietal cells form from the distal daughter cell of the mitotic division of the archesporial cell. In these cases, the MMC forms from the proximal daughter cell (Figures 9A,B). In ovules of tenuinucellate species, the opposite normally occurs, i.e., the MMC differentiates distally, and the proximal cell is considered nucellar (Johri et al., 1992). In this respect, the Boechereae show tendencies toward crassinucellate development, with parietal cells sometimes undergoing further division to produce a parietal tissue that positions the meiocyte deeper within the ovule (Figures 9C-G).

Sexual and aposporous gametophyte formation were of the eight-nucleate Polygonum type (Figures $\mathbf{9 H}-\mathbf{J}$ ). In the aposporous taxa, one or more nucellar cells, and sometimes parietal cells, initiated vacuolate gametophyte formation as early as MMC differentiation (Figures 9K,L). As described above, this may have terminated meiosis prior to $\mathrm{M}_{I I}$ and caused the abnormally high sexual dyad to tetrad ratio observed for the P. fremontii population ES 1078 (Figure 8). Nucellar, parietal, and nucellar epidermis cells degenerated quickly in front of the rapidly growing sexual or aposporous gametophytes (Figures $9 \mathrm{H}-\mathbf{N}$ ).

\section{DISCUSSION}

\section{The Ancestor of the Boechereae Had Seven Chromosomes and Descended From an ACK-Like Genome With Eight Chromosomes}

Using BAC-based CCP, we analyzed 14 samples of Boechereae representing seven of the nine genera recognized by Alexander et al. (2013). These analyses revealed a high level of genomic stasis across the tribe, with the earliest diverging genus Polyctenium (Figure 1; Beilstein et al., 2010; Couvreur et al., 2010) and B. gracilipes (a member of the derived "western Boechera clade," Figure 1) showing identical chromosome structures (Figure 2). Our analysis corroborrated our earlier hypothesis (Mandáková et al., 2015b, 2020) of an ancestral Boechereae genome with seven chromosomes $(n=7)$ derived from an older $n=8$ genome by descending dysploidy. This precursor $n=8$ genome structurally resembled the ACK, an ancestral genomic arrangement present in many tribes of crucifer lineage I (Lysak et al., 2016). Indeed, analysis of the $P$. micrantha genome from the Halimolobeae, sister group to the Boechereae, confirmed that the eight chromosomes of Halimolobeae genomes (Bailey et al., 2007) are homeologous to the eight chromosomes of the ACK. While the eight ancestral chromosomes remained conserved in Halimolobeae, the divergence of extant Boechereae appears to coincide with or follow a descending dysploidal change from $n=8$ to $n=7$. 

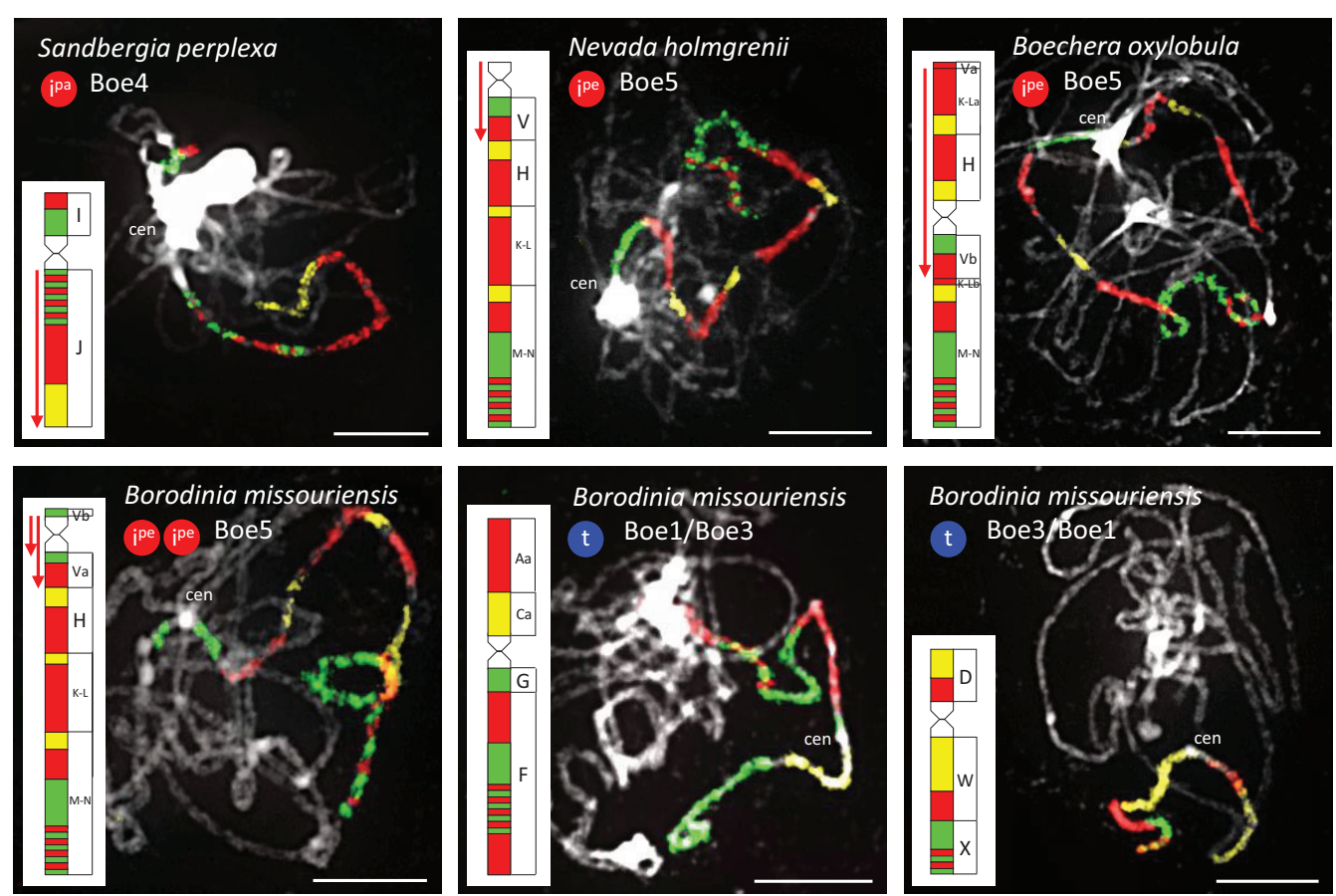

FIGURE 6 | Species- and genus-specific chromosomal rearrangements in diploid Boechereae revealed by CCP. Red downward-pointing arrows denote inverse orientation of GBs compared to their position in the ancestral Boechereae genome (Figure 3). pe: pericentric inversion; pa: paracentric inversion; t: whole-arm translocation. Chromosomes were identified by CCP with A. thaliana BAC contigs labeled by biotin-dUTP (red flourescence), digoxigenin-dUTP (green) and Сy3-dUTP (yellow). A. thaliana BAC clones defining each BAC contig/painting probe are listed in Supplementary Tables S4, S5, $\mathbf{5 7}$, S8. Chromosomes were counterstained by DAPI. Scale bars, $10 \mu \mathrm{m}$.

\section{Tribe-Specific Descending Dysploidy}

Given that all sampled Boechereae exhibit a chromosome base number of $x=7$, it is likely that the descending dysploidy event documented above occurred prior to the initial divergence of the extant members of the tribe approximately 8 million years ago (Couvreur et al., 2010). This reduction of chromosome number did not result from a "simple fusion" of two chromosomes. The first step involved the origin of a transient translocation (AK3/AK8) chromosome by a wholearm translocation, followed by an end-to-end translocation between AK3/AK8 and AK5 $(\rightarrow$ chromosomes Boe3, Boe5). The chromosome-arm collinearity of the resulting Boe5 points to inactivation or loss of the AK5 centromere. Interestingly, this paleocentromere has disappeared from many other crucifer genomes independently, and it is the most frequently inactivated centromere detected so far among the tribes of lineage I (Camelineae: Lysak et al., 2016; Cardamineae: Mandáková et al., 2016; Microlepidieae, Mandáková et al., 2010, 2017b). As tribal divergence post-dates the origin of four tribe-specific chromosomes, we propose that the three translocations involving five out of eight ancestral chromosomes were a key evolutionary innovation underlying the origin and diversification of the Boechereae in North America.

\section{Genus- and Species-Specific Inversions}

Although reciprocal translocations clearly played a major role in the origin of the tribe, the only additional translocation noted within the tribe was a unique whole-arm transfer between Boe1 and Boe3 that produced two structurally unique chromosomes in B. missouriensis (Figure 2). However, chromosomal inversions proved to be common within the group, as they are in other mustards (e.g., Mandáková et al., 2015a; Fransz et al., 2016) and land plants in general (Hoffmann and Rieseberg, 2008). Autapomorphic pericentric inversions on chromosome Boe5 were observed in both Borodinia and Nevada, while Cusickiella exhibited unique pericentric and paracentric inversions on Boe4 (Figure 2).

Our analyses identified three potentially synapomorphic chromosomal rearrangements within the tribe: (1) a 9.83$\mathrm{Mb}$ pericentric inversion on Boe5 shared by $B$. formosa and B. oxylobula, but not by B. gracilipes, (2) a $2.55-\mathrm{Mb}$ pericentric inversion on Boe5 shared by $N$. holmgrenii and Borodinia missouriensis, and (3) a 8.24-Mb paracentric inversion on Boe4 shared by $N$. holmgrenii, all three ploidies of $P$. cheiranthoides, and both species of Sandbergia (Figure 2). Support for the synapomorphic status of these three chromosomal rearrangements is equivocal in the few phylogenetic analyses of Boechereae published to date.

With respect to the $9.83-\mathrm{Mb}$ pericentric inversion apparently shared by B. formosa and B. oxylobula, the only phylogenetic analysis with appropriate taxon sampling is the concatenated nuclear gene tree of Alexander et al. (2013). This tree shows very weak support for a clade encompassing $B$. oxylobula and $B$. gracilipes but excluding B. formosa. A close relationship 


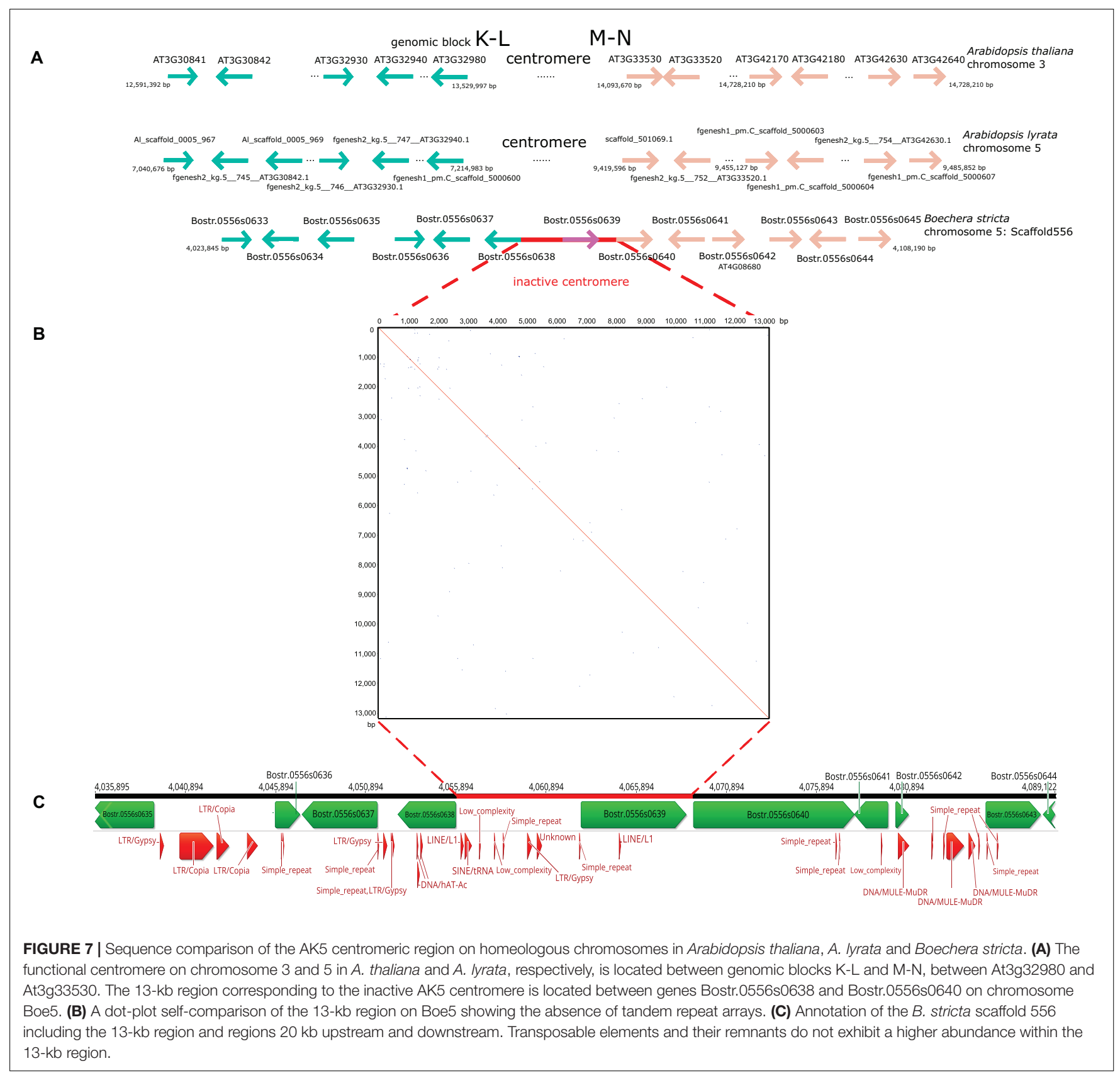

between B. oxylobula (which has the inversion) and B. gracilipes (which does not) is congruent with morphology in this case, and it argues against the $9.83-\mathrm{Mb}$ pericentric inversion being synapomorphic.

Concerning the $2.55-\mathrm{Mb}$ pericentric inversion shared by $N$. holmgrenii and B. missouriensis, the two published analyses with appropriate taxon sampling yielded conflicting topologies. The concatenated nuclear gene tree of Alexander et al. (2013) provides very weak support for a sister relationship between Nevada and Borodinia, which would favor the interpretation of the 2.55-Mb inversion as a synapomorphic character. However, the Boechereae phylogeny presented by Beilstein et al. (2010) places Nevada as sister to Phoenicaulis not Borodinia, the latter being sister to a clade comprising Boechera s.s. and Anelsonia (Figure 1B). If this phylogeny is correct, the $2.55-\mathrm{Mb}$ inversion apparently shared by Nevada and Borodinia would have originated independently.

The final potential chromosomal synapomorphy to be considered is the $8.24-\mathrm{Mb}$ paracentric inversion shared by $N$. holmgrenii, all three ploidies of $P$. cheiranthoides, and both species of Sandbergia (Figure 2). Among previously published phylogenies, the Beilstein et al. (2010) topology hypothesizes a sister relationship between Nevada and Phoenicaulis (Sandbergia was not sampled). This would be congruent with the $8.24-\mathrm{Mb}$ 


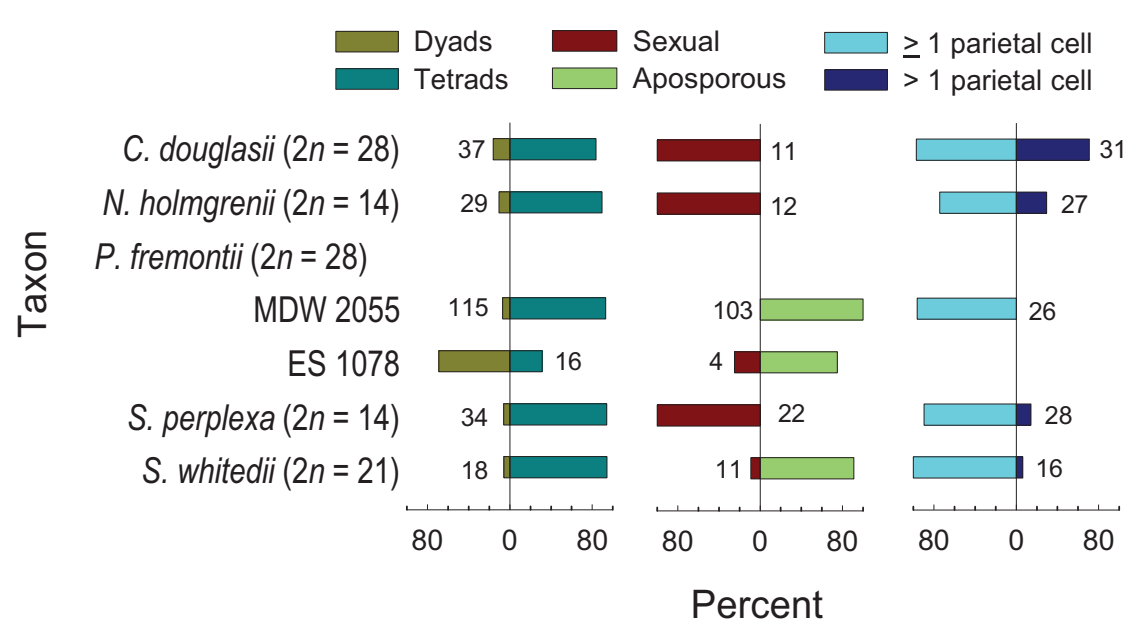

FIGURE 8 | Frequencies of dyads vs. tetrads, sexual vs. aposporous gametophytes, and parietal cells or tissues ( $\geq 1$ parietal cell) vs. parietal tissues (>1 parietal cell). Numbers next to bars represent observations contributing to each variable pair.

inversion on Boe4 being interpreted as synapomorphic. However, the concatenated nuclear gene tree of Alexander et al. (2013) hypothesizes a sister relationship (very weakly supported) between Nevada and Borodinia and provides no resolution of inter-generic relationships for Phoenicaulis or Sandbergia. This creates a conflict between the only two inversions that could be a synapomorphy. If the Beilstein et al. (2010) phylogeny is correct, then the $8.24-\mathrm{Mb}$ inversion discussed here could be synapomorphic but the $2.55-\mathrm{Mb}$ inversion mentioned previously would not. On the other hand, if the Alexander et al. (2013) topology is correct, then the 2.55-Mb inversion could be synapomorphic but the $8.24-\mathrm{Mb}$ inversion would not. A better resolved and supported phylogeny will be needed to assess the homology of the chromosomal rearrangements documented herein.

Chromosomal inversions appear to be relatively common in Boechereae, but the existence of breakage "hotspots" on several chromosomes can make it difficult to infer homology. In our dataset, most of the inversions detected are pericentric and occur in just one or two samples or taxa. The only inversion that appears to have any time depth is the $8.24-\mathrm{Mb}$ paracentric inversion on Boe4 (shared by six samples representing four species and three genera). The others appear to be more recent, like the 8.4$\mathrm{Mb}$ paracentric inversion on chromosome Bs1 that distinguishes the West genotype of $B$. stricta from other populations of the species (Lee et al., 2017). Some of these young inversions likely originated since the last glacial maximum, suggesting that this type of chromosomal rearrangement may be an ongoing and important contributor to reproductive isolation and speciation within the Boechereae.

\section{The Boechereae $\boldsymbol{n}=\mathbf{8}$ Ancestor in a Phylogeographic Context}

Eurasia, specifically the Irano-Turanian floristic region, is believed to be the cradle of crucifer origin (Franzke et al., 2009). Although long-distance dispersal events contributed to plant migrations from Eurasia to North America and in the opposite direction (e.g., Wang et al., 2015; Huang et al., 2018), the Bering land bridge played the key role for the spreading of seed plants, including crucifers, from Eurasia to the North American subcontinent (e.g., Carlsen et al., 2010; Wen et al., 2010; Karl and Koch, 2013; Jiang et al., 2019). Among the clades belonging to crucifer lineage I, some are endemic to the New World (Halimolobeae and Physarieae), others have a bi-continental distribution in Asia and America (Camelineae, Cardamineae, Crucihimalayeae, Descurainieae, Erysimeae, and Smelowskieae), but the Boechereae are confined to North America with only three species occurring in the Russian Far East. The bi-continental distribution of several tribes in the lineage I, and tribes of other crucifer lineages (e.g., Arabideae: Karl and Koch, 2013), makes the Bering land bridge the most plausible colonization route for several (and perhaps all) crucifer clades to North America.

Since the Halimolobeae are consistently retrieved as the sister clade to Boechereae (e.g., Beilstein et al., 2010; Nikolov et al., 2019) and Halimolobeae genomes resemble the ACK genome with $n=8$, it is likely that the ancestral genomes of Boechereae and Halimolobeae arose from this common ancestor. The present-day geographic ranges of the two tribes differ, with Boechereae concentrated in the United States and Canada (barely extending into Mexico) and Halimolobeae generally more southern in distribution. In fact, Halimolobeae has two distinct centers of diversity, one extending from central Mexico to the southwestern United States, and the other in the Andes, from Ecuador to central Argentina (Bailey et al., 2007). The geographic ranges of the two tribes overlap in a narrow band stretching east to west along the United States/Mexico border. As both tribes have the closest phylogenetic affinity to the primarily Eurasian clades of lineage I, we propose that the MRCA of Boechereae/Halimolobeae reached North America via the Bering land bridge. Couvreur et al. (2010) estimated that the Boechereae and Halimolobeae diverged c. 8 mya (late Miocene) and their 


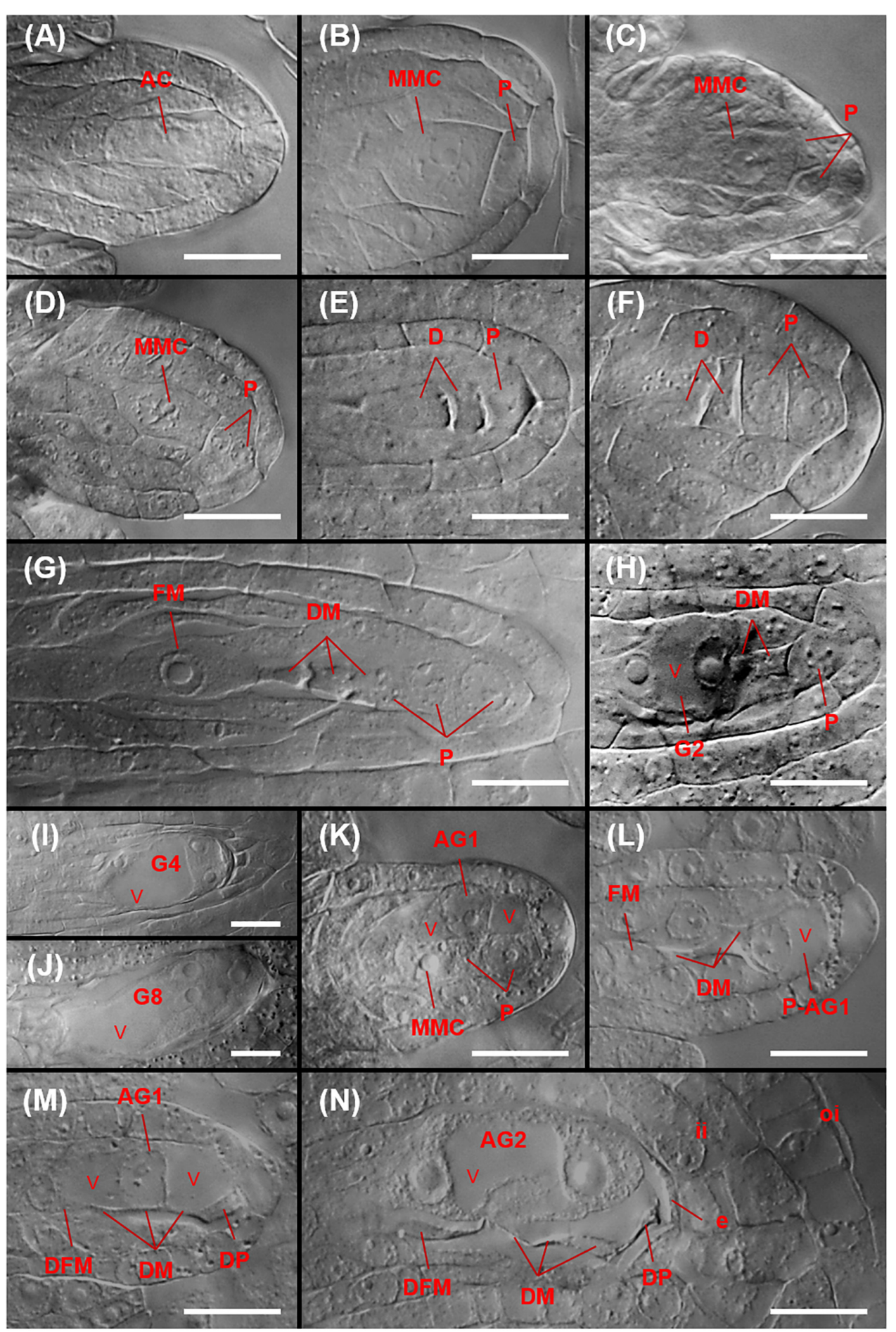

FIGURE 9 | Megasporogenesis and sexual and aposporous gametophyte formation in P. fremontii (A,K-N) and sexual C. douglasii (B,D,F,G), N. holmgrenii (C), and S. perplexa (E,H-J). (A) Archesporial cell (AC) at the budding integument stage. (B) Mitotic division of an AC yielding a proximal MMC and a distal parietal cell (P). (C,D) Anticlinal and paraclinal divisions, respectively, of a P to yield a two-celled parietal tissue. (E,F) Dyads (D) with one and two Ps, respectively. (G) Tetrad showing the functional megaspore (FM) and three degenerating megaspores (DM). Also shown is a parietal tissue consisting of three Ps that formed from two paraclinal divisions of the original P. (H) Two-nucleate sexual gametophyte (G2) showing a central vacuole (V), two of three DM, and a P. (I) Four-nucleate sexual gametophyte (G4) with three nuclei visible. (J) Eight-nucleate sexual gametophyte (G8) showing egg apparatus formation at the micropylar end of the gametophyte. (K) 1-nucleate aposporous gametophyte (AG1) from a nucellar cell at the MMC stage. (L) AG1 from a parietal cell (P-AG1) at the tetrad stage. (M) AG1 from a nucellar cell at the late tetrad stage showing functional megaspore degeneration (DFM), DMs, degenerating nucellar cells, and a degenerating parietal cell (DP). (N) Two-nucleate aposporous gametophyte (AG2) at the late tetrad stage showing a DFM, DMs, degenerating nucellar cells, a degenerating parietal cell (DP), and degenerating epidermal cells. Scale bars, $20 \mu \mathrm{m}$. 
MRCA diverged from its predominantly Eurasian sister lineage during mid to early Miocene. The Bering land bridge connected northeastern Asia and northwestern North America from the Cretaceous until the Pliocene (Gladenkov et al., 2002; Jiang et al., 2019). In the latter study, rates of dispersal from Eurasia to North America were significantly elevated throughout the Oligocene and early Miocene (c. 34 to 16 mya), particularly around 26 to 24 mya. These time estimates broadly coincide with the origin and diversification of Brassicaceae lineage I in early Miocene ( 23 to 18 mya; Hohmann et al., 2015), which likely used the Bering land bridge to disperse from northeastern Asia to North America.

\section{Apomixis-Related Chromosomes in the Boechereae}

Two heterochromatic chromosomes (Het and Del) have been previously described in Boechera apomicts (Kantama et al., 2007; Mandáková et al., 2015a). In eudiploid apomicts $(2 n=14)$, a Het chromosome was identified as one of the Boel homologs (GBs A, C, and D). In aneuploid apomicts $(2 n=15,22)$, a centric fission partitioned Het chromosome to a larger Het (GBs A and C) and to a smaller $\mathrm{Del}$ (block D) chromosome (Mandáková et al., 2015b). In P. cheiranthoides, apomictic triploids and tetraploids contained a heterochromatic Het (GBs A, C, and D), but it was absent in diploid apomicts (Mandáková et al., 2020). This observation indicates that aposporic reproduction in Phoenicaulis is not associated with the presence of a Het chromosome. This is further corroborated herein by the apparent absence of a Het chromosome in two other aposporous apomicts, tetraploid $P$. fremontii and triploid S. whitedii.

\section{Autotetraploids in the Boechereae}

While diploid or nearly diploid $(2 n=15)$ species and hybrids seem to prevail in Boechereae (cf. BrassiBase ${ }^{4}$, accessed on 1 February 2020), apomictic triploid $(2 n=21)$ or nearly triploid $(2 n=22)$ hybrids are very common in Boechera (Schranz et al., 2005; Li et al., 2017) and recently discovered in Phoenicaulis (Mandáková et al., 2020) and S. whitedii (this study). Tetraploids $(2 n=28)$ are rarely reported and in Boechera all cases of tetraploidy studied to date involve interspecific hybridization (i.e., allopolyploidy; see Windham et al., 2014; Li et al., 2017). Autotetraploidy was previously documented only in P. cheiranthoides (Mandáková et al., 2020), where the tetraploid cytotype is more common and widespread than either the diploid or triploid. Here we report two new cases of autotetraploidy in Boechereae, involving C. douglasii and P. fremontii. Hence, bona fide autotetraploids occur in three out of nine Boechereae genera. The autotetraploid species/cytotypes reproduce either sexually (Cusickiella) or, more frequently, by apomixis (Phoenicaulis and Polyctenium). Autopolyploids often experience irregular chromosome segregation due to the multivalent formation, but apomixis can potentially bypass such problematic meioses (Darlington, 1939; Stebbins, 1971; Comai, 2005; Cosendai et al., 2011). Indeed, while the analyzed tetraploid plants of P. fremontii show exclusive quadrivalent pairing (Figure 5), they

${ }^{4}$ https://brassibase.cos.uni-heidelberg.de/ appear to be fully fertile due to apospory (Figure 8). Hence, apomixis appears to be stabilizing reproduction in triploids and autotetraploids, which otherwise would suffer from semi-sterility due to chromosome pairing irregularities.

\section{Apomixis Originated Several Times Independently During Brassicaceae and Boechereae Diversification}

Following the pioneering work on apomixis in B. holboellii [as Arabis holboellii Hornem. in Böcher (1951)], Mulligan (1966) reported its occurrence in Erysimum L. (Erysimeae) and Mosquin and Hayley (1966) documented possible asexual seed production in Parrya R. Brown (Chorisporeae). Mulligan and Findlay (1970) identified several species of Draba L. (Arabideae) that they inferred to be apomictic, and subsequent embryological and single seed flow cytometry analyses of one of these species (Draba oligosperma Hook.) were suggestive of apospory (JordonThaden and Koch, 2012). Detailed cytological studies of Draba, Erysimum, and Parrya are needed to verify the regular occurrence of asexual seed production and the specific pathway involved. However, the available anecdotal evidence for this reproductive pathway occurring in four distantly related crucifer tribes (and its induction by hybridization in a fifth tribe, Brassiceae; Ellerstrom, 1983) suggests that apomixis has evolved independently multiple times within the family.

The Boechereae appear to be a "hotspot" for the origin and diversification of apomictic taxa. Our earlier publications have embryologically confirmed apomixis (either diplospory or apospory) in more than 20 diploid and triploid taxa of Boechera (Windham et al., 2015; Carman et al., 2019), and apospory in diploid, triploid, and tetraploid Phoenicaulis (Mandáková et al., 2020), and in diploid Borodinia laevigata (Muhl. ex Willd.) P. J. Alexander and Windham [as Boechera laevigata (Muhl. ex Willd.) Al-Shehbaz in Carman et al., 2019]. Here, two new genera are added to the list of aposporous apomicts, Polyctenium (tetraploid) and Sandbergia (triploid). Two genera of Boechereae (Anelsonia and Yosemitea) remain unsampled, and the exclusive occurrence of sexual reproduction in Nevada and Cusickiella must be confirmed by more extensive sampling. In summary, apomixis is now known to occur in five of the nine genera of Boechereae, and in two of these (Phoenicaulis and Polyctenium) it is the only reproductive pathway documented to date. Based on current sampling, Sandbergia exhibits equal proportions of apomictic (S. whitedii) and sexual reproduction (S. perplexa). Among Boechera species, apomictic taxa of hybrid origin greatly outnumber their sexual diploid progenitors (Li et al., 2017), while in Borodinia, sexual populations appear to predominate.

High frequency apospory ( $70 \%$ of ovules) was observed in man-made Raphanus L. $\times$ Brassica L. hybrids (Brassiceae) (Ellerstrom and Zagorcheva, 1977; Ellerstrom, 1983), which is consistent with wide hybridization occasionally inducing apomixis in otherwise sexual species (Carman, 1997). The longterm reproductive stability conferred by apomixis to sterile or semisterile inter-specific hybrids could provide novel genotypes with sufficient time (possibly hundreds of years) to fortuitously produce, by facultative sexual reproduction, recombinants that 
are more or less genomically stable (autopolyploidized, sensu Sybenga, 1996) and sexually fertile (Carman, 1997, 2007; Carman et al., 2019). If chromosome aberrations have occurred, the newly formed recombinant genomes might warrant specific or generic status. Hence, apomixis in genomically unstable taxa may facilitate (Carman, 1997; Horandl and Hojsgaard, 2012; Hojsgaard et al., 2014; Carman et al., 2019) rather than terminate (Darlington, 1939; Stebbins, 1971) speciation. That apomixis is prevalent in many large genera, e.g., among the rose, aster, and grass families (Carman, 1997; Hojsgaard et al., 2014), as well as in Boechera, supports this hypothesis.

\section{DATA AVAILABILITY STATEMENT}

All datasets generated for this study are included in the article/Supplementary Material.

\section{AUTHOR CONTRIBUTIONS}

TM, JC, and ML conceived the experiments. TM-O, PH, KA, and BP conducted the study and processed the data. ML, TM, JC, and MW wrote the manuscript. All authors have read and approved the final manuscript.

\section{REFERENCES}

Alexander, P. J., Windham, M. D., Beck, J. B., Al-Shehbaz, I. A., Allphin, L., and Bailey, C. D. (2013). Molecular phylogenetics and taxonomy of the genus Boechera and related genera (Brassicaceae: Boechereae). Syst. Bot. 38, 192-209. doi: 10.1600/036364413x661917

Alexander, P. J., Windham, M. D., Beck, J. B., Al-Shehbaz, I. A., Allphin, L., and Bailey, C. D. (2015). Weaving a tangled web: divergent and reticulate speciation in Boecher fendleri sensu lato (Brassicaceae; Boechereae). Syst. Bot. 40, 572-596. doi: $10.1600 / 036364415 \times 688745$

Al-Shehbaz, I. A. (2012). A generic and tribal synopsis of the Brassicaceae (Cruciferae). Taxon 61, 931-954. doi: 10.1002/tax.615002

Asker, S. E., and Jerling, L. (1992). Apomixis in Plants. Boca Raton, FL: CRC Press.

Bailey, C. D., Al-Shehbaz, I. A., and Rajanikanth, G. (2007). Generic limits in tribe Halimolobeae and description of the new genus Exhalimolobos (Brassicaceae). Syst. Bot. 32, 140-156. doi: 10.1600/036364407780360166

Beck, J. B., Alexander, P. J., Allphin, L., Al-Shehbaz, I. A., Rushworth, C., Bailey, C. D., et al. (2012). Does hybridization drive the transition to asexuality in diploid Boechera (Brassicaceae)? Evolution 66, 985-995. doi: 10.1111/j.15585646.2011.01507.x

Beilstein, M. A., Nagalingum, N. S., Clements, M. D., Manchester, S. R., and Mathews, S. (2010). Dated molecular phylogenies indicate a Miocene origin for Arabidopsis thaliana. Proc. Natl. Acad. Sci. U.S.A. 107, 18724-18728. doi: 10.1073/pnas.0909766107

Böcher, T. W. (1951). Cytological and embryological studies in the amphiapomictic Arabis holboellii complex. Det Kongelige Danske Videnskabernes Selskab. Biol. Skrif. 6, 1-59.

Brukhin, V., Osadtchiy, J. V., Florez-Rueda, A. M., Smetanin, D., Bakin, E., Nobre, M. S., et al. (2019). The Boechera genus as a resource for apomixis research. Front. Plant Sci. 10:392. doi: 10.3389/fpls.2019.00392

Carlsen, T., Elven, R., and Brochmann, C. (2010). The evolutionary history of Beringian Smelowskia (Brassicaceae) inferred from combined microsatellite and DNA sequence data. Taxon 59, 427-438. doi: 10.1002/tax.592008

Carman, J. G. (1997). Asynchronous expression of duplicate genes in angiosperms may cause apomixis, bispory, tetraspory, and polyembryony. Biol. J. Linn. Soc. 61, 51-94. doi: 10.1111/j.1095-8312.1997.tb01778.x

\section{FUNDING}

This work was supported by Czech Ministry of Education, Youth and Sports within the program INTER-EXCELLENCE (Project No. LTAUSA17002), by the CEITEC 2020 project (Grant No. LQ1601), the Utah Agricultural Experiment Station project awards, Utah State University, Logan to JC, and the U.S. National Science Foundation (award DEB-0816560 to MW) and the U.S. National Institutes of Health (Grant R01 GM086496 to TM-O).

\section{ACKNOWLEDGMENTS}

We thank Donovan Bailey, Eric Schranz, and Millenium Seed Bank (Royal Botanic Gardens, Kew) for providing seeds, and Dmitry German for his comments on the manuscript. Core Facility Plants Sciences of CEITEC MU is acknowledged for the cultivation of plants used in this manuscript.

\section{SUPPLEMENTARY MATERIAL}

The Supplementary Material for this article can be found online at: https://www.frontiersin.org/articles/10.3389/fpls.2020.00514/ full\#supplementary-material

Carman, J. G. (2007). “Do duplicate genes cause apomixis?”” in Apomixis: Evolution, Mechanisms and Perspectives, eds E. Horandl, U. Grossniklaus, P. J. van Dijk, and T. F. Sharbel (Liechtenstein: A. R. G. Gantner Verlag K. G), 63-91.

Carman, J. G., Mateo de Arias, M., Gao, L., Zhao, X., Kowallis, B., Sherwood, D. A., et al. (2019). Apospory in addition to diplospory is common in Boechera where it may facilitate speciation by recombination-driven apomixis-to-sex reversals. Front. Plant Sci. 10:724. doi: 10.3389/fpls.2019.00724

Carter, K. A., Liston, A., Bassil, N. V., Alice, L. A., Bushakra, J. M., Sutherland, B. L., et al. (2019). Target capture sequencing unravels Rubus evolution. Front. Plant Sci. 10:1615. doi: 10.3389/fpls.2019.01615

Cheng, F., Wu, J., Fang, L., and Wang, X. (2012). Syntenic gene analysis between Brassica rapa and other Brassicaceae species. Front. Plant Sci. 3:198. doi: 10. 3389/fpls.2019.00198

Comai, L. (2005). The advantages and disadvantages of being polyploid. Nat. Rev. Genet. 6, 836-846. doi: 10.1038/nrg1711

Cosendai, A. C., Rodewald, J., and Hörandl, E. (2011). Origin and distribution of autopolyploids via apomixis in the alpine species Ranunculus kuepferi (Ranunculaceae). Taxon 60, 355-364. doi: 10.1002/tax.602006

Couvreur, T. L., Franzke, A., Al-Shehbaz, I. A., Bakker, F. T., Koch, M. A., and Mummenhoff, K. (2010). Molecular phylogenetics, temporal diversification, and principles of evolution in the mustard family (Brassicaceae). Mol. Biol. Evol. 27, 55-71. doi: 10.1093/molbev/msp202

Cowie, R. H., and Holland, B. S. (2008). Molecular biogeography and diversification of the endemic terrestrial fauna of the Hawaiian Islands. Philos. Trans. R. Soc. Lond. B Biol. Sci. 363, 3363-3376. doi: 10.1098/rstb.2008.0061

Darlington, C. D. (1939). The Evolution Of Genetic Systems. Cambridge: Cambridge University Press.

Doudkin, R. V., and Volkova, S. A. (2013). A new species of Boechera (Brassicaceae) from the Primorsky Territory, Russia. Novon 22, 411-414. doi: 10.3417/ 2010077

Ellerstrom, S. (1983). Apomictic progeny from Raphanobrassica. Hereditas 99:315. doi: 10.1111/j.1601-5223.1983.tb00906.x

Ellerstrom, S., and Zagorcheva, L. (1977). Sterility and apomictic embryo-sac formation in Raphanobrassica. Hereditas 87, 107-119. doi: 10.1111/j.16015223.1977.tb01251.x 
Faria, R., and Navarro, A. (2010). Chromosomal speciation revisited: rearranging theory with pieces of evidence. Trends Ecol. Evol. 25, 660-669. doi: 10.1016/j. tree.2010.07.008

Fransz, P., Linc, G., Lee, C. R., Aflitos, S. A., Lasky, J. R., Toomajian, C., et al. (2016). Molecular, genetic and evolutionary analysis of a paracentric inversion in Arabidopsis thaliana. Plant J. 88, 159-178. doi: 10.1111/tpj.13262

Franzke, A., German, D., Al-Shehbaz, I. A., and Mummenhoff, K. (2009). Arabidopsis family ties: molecular phylogeny and age estimates in Brassicaceae. Taxon 58, 425-437. doi: 10.1002/tax.582009

Franzke, A., Lysak, M. A., Al-Shehbaz, I. A., Koch, M. A., and Mummenhoff, K. (2011). Cabbage family affairs: the evolutionary history of Brassicaceae. Trends Plant Sci. 16, 108-116. doi: 10.1016/j.tplants.2010.11.005

Freyman, W. A., and Höhna, S. (2017). Cladogenetic and anagenetic models of chromosome number evolution: a Bayesian model averaging approach. Syst. Biol. 67, 195-215. doi: 10.1093/sysbio/syx065

German, D. A., and Friesen, N. W. (2014). Shehbazia (Shehbazieae, Cruciferae), a new monotypic genus and tribe of hybrid origin from Tibet. Turczaninowia 17, 17-23. doi: 10.14258/turczaninowia.17.4.3

Givnish, T. J., Spalink, D., Ames, M., and Lyon, S. P. (2016). Orchid historical biogeography, diversification, Antarctica and the paradox of orchid dispersal. J. Biogeogr. 43, 1905-1916. doi: 10.1111/jbi.12854

Gladenkov, A. Y., Oleinik, A. E., and Marincovich, L. (2002). A refined age for the earliest opening of Bering strait. Palaeogeogr. Palaeoclimatol. Palaeoecol. 183, 321-328. doi: 10.1016/s0031-0182(02)00249-3

Hand, M. L., and Koltunow, A. M. (2014). The genetic control of apomixis: asexual seed formation. Genetics 197, 441-450. doi: 10.1534/genetics.114.163105

Hoffmann, A. A., and Rieseberg, L. H. (2008). Revisiting the impact of inversions in evolution: from population genetic markers to drivers of adaptive shifts and speciation? Annu. Rev. Ecol. Evol. Syst. 39, 21-42. doi: 10.1146/annurev.ecolsys. 39.110707.173532

Hohmann, N., Wolf, E. M., Lysak, M. A., and Koch, M. A. (2015). A time-calibrated road map of Brassicaceae species radiation and evolutionary history. Plant Cell 27, 2770-2784. doi: 10.1105/tpc.15.00482

Hojsgaard, D., Klatt, S., Baier, R., Carman, J. G., and Horandl, E. (2014). Taxonomy and biogeography of apomixis in angiosperms and associated biodiversity characteristics. Crit. Rev. Plant Sci. 33, 414-427. doi: 10.1080/07352689.2014. 898488

Horandl, E., and Hojsgaard, D. (2012). The evolution of apomixis in angiosperms: A reappraisal. Plant Biosyst. 146, 681-693.

Hu, T. T., Pattyn, P., Bakker, E. G., Cao, J., Cheng, J. F., Clark, R. M., et al. (2011). The Arabidopsis lyrata genome sequence and the basis of rapid genome size change. Nat. Genet. 43, 476-481. doi: 10.1038/ng.807

Huang, C. H., Sun, R., Hu, Y., Zeng, L., Zhang, N., Cai, L., et al. (2016). Resolution of Brassicaceae phylogeny using nuclear genes uncovers nested radiations and supports convergent morphological evolution. Mol. Biol. Evol. 33, 394-412. doi: 10.1093/molbev/msv226

Huang, J., Yang, L. Q., Yu, Y., Liu, Y. M., Xie, D. F., Li, J., et al. (2018). Molecular phylogenetics and historical biogeography of the tribe Lilieae (Liliaceae): bidirectional dispersal between biodiversity hotspots in Eurasia. Ann. Bot. 122, 1245-1262. doi: 10.1093/aob/mcy138

Jiang, D., Klaus, S., Zhang, Y.-P., Hillis, D. M., and Li, J.-T. (2019). Asymmetric biotic interchange across the Bering land bridge between Eurasia and North America. Nat. Sci. Rev. 6, 739-745. doi: 10.1093/nsr/nwz035

Johri, B. M., Ambegaokar, K. B., and Srivastava, P. S. (1992). Comparative Embryology of Angiosperms, Vol. 1. New York, NY: Springer-Verlag.

Jordon-Thaden, I., and Koch, M. A. (2012). "Detection of apomixis in an octoploid, alpine species, Draba oligosperma (Brassicaceae)," in Proceedings of the International Plant and Animal Genome Conference XX, San Diego, CA.

Jordon-Thaden, I. E., Al-Shehbaz, I. A., and Koch, M. A. (2013). Species richness of the globally distributed, arctic-alpine genus Draba L. (Brassicaceae). Alpine Bot. 123, 97-106. doi: 10.1007/s00035-013-0120-9

Kantama, L., Sharbel, T. F., Schranz, M. E., Mitchell-Olds, T., de Vries, S., and de Jong, H. (2007). Diploid apomicts of the Boechera holboellii complex display large-scale chromosome substitutions and aberrant chromosomes. Proc. Natl. Acad. Sci. U.S.A. 104, 14026-14031. doi: 10.1073/pnas.0706647104

Karl, R., and Koch, M. A. (2013). A world-wide perspective on crucifer speciation and evolution: phylogenetics, biogeography and trait evolution in tribe Arabideae. Ann. Bot. 112, 983-1001. doi: 10.1093/aob/mct165
Kim, C., Kim, S.-C., and Kim, J.-H. (2019). Historical biogeography of melanthiaceae: a case of Out-of-North America through the bering land Bridge. Front. Plant Sci. 10:396. doi: 10.3389/fpls.2019.00396

Kliver, S., Rayko, M., Komissarov, A., Bakin, E., Zhernakova, D., Prasad, K., et al. (2018). Assembly of the Boechera retrofracta genome and evolutionary analysis of apomixis-associated genes. Genes 9:185. doi: 10.3390/genes 9040185

Koch, M. A., Haubold, B., and Mitchell-Olds, T. (2000). Comparative evolutionary analysis of chalcone synthase and alcohol dehydrogenase loci in Arabidopsis, Arabis, and related genera (Brassicaceae). Mol. Biol. Evol. 17, 1483-1498. doi: 10.1093/oxfordjournals.molbev.a026248

Lee, C. R., Wang, B., Mojica, J. P., Mandáková, T., Prasad, K. V. S. K., Goicoechea, J. L., et al. (2017). Young inversion with multiple linked QTLs under selection in a hybrid zone. Nat. Ecol. Evol. 1:119.

Li, F. W., Rushworth, C. A., Beck, J. B., and Windham, M. D. (2017). Boechera microsatellite website: an online portal for species identification and determination of hybrid parentage. Database 2017:baw169. doi: 10.1093/ database/baw169

Linder, H. P., and Barker, N. P. (2014). Does polyploidy facilitate long-distance dispersal? Ann. Bot. 113, 1175-1183. doi: 10.1093/aob/mcu047

Lysak, M. A., and Mandáková, T. (2013). “Analysis of plant meiotic chromosomes by chromosome painting," in Methods in molecular biology, ed. N. J. Clifton (New York, NY: Humana Press), 13-24. doi: 10.1007/978-1-62703-333-6_2

Lysak, M. A., Mandáková, T., and Schranz, M. E. (2016). Comparative paleogenomics of crucifers: ancestral genomic blocks revisited. Curr. Opin. Plant Biol. 30, 108-115. doi: 10.1016/j.pbi.2016.02.001

Mandáková, T., and Lysak, M. A. (2008). Chromosomal phylogeny and karyotype evolution in $x=7$ crucifer species (Brassicaceae). Plant Cell 20, 2559-2570. doi: 10.1105/tpc.108.062166

Mandáková, T., and Lysak, M. A. (2016a). Chromosome preparation for cytogenetic analyses in Arabidopsis. Curr. Protoc. Plant Biol. 1, 43-51. doi: 10.1002/cppb.20009

Mandáková, T., and Lysak, M. A. (2016b). Painting of Arabidopsis chromosomes with chromosome-specific BAC clones. Curr. Protoc. Plant Biol. 1, 359-371. doi: $10.1002 / \mathrm{cppb} .20022$

Mandáková, T., Ashby, K., Price, B. J., Windham, M. D., Carman, J. G., and Lysak, M. A. (2020). Genome structure and apomixis in Phoenicaulis (Brassicaceae; Boechereae). J. Syst. Evol. doi: 10.1111/jse.12555

Mandáková, T., Gloss, A. D., Whiteman, N. K., and Lysak, M. A. (2016). How diploidization turned a tetraploid into a pseudotriploid. Am. J. Bot. 103, 1-10.

Mandáková, T., Heenan, P. B., and Lysak, M. A. (2010). Island species radiation and karyotypic stasis in Pachycladon allopolyploids. BMC Evol. Biol. 10:367. doi: 10.1186/1471-2148-10-367

Mandáková, T., Hloušková, P., German, D. A., and Lysak, M. A. (2017a). Monophyletic origin and evolution of the largest crucifer genomes. Plant Physiol. 174, 2062-2071. doi: 10.1104/pp.17.00457

Mandáková, T., Pouch, M., Harmanová, K., Zhan, S. H., Mayrose, I., and Lysak, M. A. (2017b). Multispeed genome diploidization and diversification after an ancient allopolyploidization. Mol. Ecol. 26, 6445-6462. doi: 10.1111/mec.14379

Mandáková, T., Schranz, M. E., Sharbel, T. F., de Jong, H., and Lysak, M. A. (2015a). Karyotype evolution in apomictic Boechera and the origin of the aberrant chromosomes. Plant J. 82, 785-793. doi: 10.1111/tpj.12849

Mandáková, T., Singh, V., Krämer, U., and Lysak, M. A. (2015b). Genome structure of the heavy metal hyperaccumulator Noccaea caerulescens and its stability on metalliferous and nonmetalliferous soils. Plant Physiol. 169, 674-689. doi: 10.1104/pp.15.00619

Mosquin, T., and Hayley, D. E. (1966). Chromosome numbers and taxonomy of some Canadian Arctic plants. Can. J. Bot. 44, 1209-1218. doi: 10.1139/b66-132

Mulligan, G. A. (1966). Chromosome numbers of the family Crucifereae III. Can. J. Bot. 44, 309-319. doi: 10.1139/b66-037

Mulligan, G. A., and Findlay, J. N. (1970). Sexual reproduction and agamospermy in the genus Draba. Can. J. Bot. 48, 269-271.

Naumova, T. N., van der Laak, J., Osadtchiy, J., Matzk, F., Kravtchenko, A., Bergervoet, J., et al. (2001). Reproductive development in apomictic populations of Arabis holboellii (Brassicaceae). Sex. Plant Reprod. 14, 195-200. doi: 10.1007/s00497-001-0118-0

Nikolov, L. A., Shushkov, P., Nevado, B., Ga, X., Al-Shehbaz, I. A., Filatov, D., et al. (2019). Resolving the backbone of the Brassicaceae phylogeny for investigating trait diversity. New Phytol. 222, 1638-1651. doi: 10.1111/nph.15732 
Ogutcen, E., and Vamosi, J. C. (2016). phylogenetic study of the tribe Antirrhineae: genome duplications and long-distance dispersals from the old world to the new world. Am. J. Bot. 103, 1071-1081. doi: 10.3732/ajb.1500464

Rojek, J., Kapusta, M., Kozieradzka-Kiszkurno, M., Majcher, D., Gorniak, M., Sliwinska, E., et al. (2018). Establishing the cell biology of apomictic reproduction in diploid Boechera stricta (Brassicaceae). Ann. Bot. 122, 513-539. doi: $10.1093 / \mathrm{aob} / \mathrm{mcy} 114$

Rokas, A., and Holland, P. W. (2000). Rare genomic changes as a tool for phylogenetics. Trends Ecol. Evol. 15, 454-459. doi: 10.1016/s0169-5347(00) 01967-4

Roy, B. A. (1995). The breeding systems of six species of Arabis (Brassicaceae). Am. J. Bot. 82, 869-877. doi: 10.1002/j.1537-2197.1995.tb15703.x

Schranz, M. E., Dobes, C., Koch, M. A., and Mitchell-Olds, T. (2005). Sexual reproduction, hybridization, apomixis, and polyploidization in the genus Boechera (Brassicaceae). Am. J. Bot. 92, 1797-1810. doi: 10.3732/ajb.92.11.1797

Stebbins, G. L. (1971). Chromosomal Evolution in Higher Plants. London: E. Arnold.

Sybenga, J. (1996). Chromosome pairing affinity and quadrivalent formation in polyploids: do segmental allopolyploids exist? Genome 39, 1176-1184. doi: 10.1139/g96- 148

Wang, X.-J., Shi, D.-C., Wang, X.-Y., Wang, J., Sun, Y.-S., and Liu, J.-Q. (2015). Evolutionary migration of the disjunct salt cress eutrema salsugineum (= Thellungiella salsuginea, Brassicaceae) between Asia and North America. PLoS One 10:e0124010. doi: 10.1371/journal.pone.0124010

Warwick, S. I., and Al-Shehbaz, I. A. (2006). Brassicaceae: chromosome number index and database on CD-Rom. Pl. Syst. Evol. 259, 237-248. doi: 10.1007/ s00606-006-0421-1
Wen, J., Ickert-Bond, S., Nie, Z. L., and Li, R. (2010). "Timing and modes of evolution of eastern Asian - North American biogeographic disjunctions in seed plants," in Darwin's Heritage Today: Proceedings of the Darwin 2010 Beijing International Conference, eds M. Long, H. Gu, and Z. Zhou (Beijing: Higher Education Press), 252-269.

Windham, M. D., Beck, J., Alexander, P., Li, F.-W., Rushworth, C., Bailey, C. D., et al. (2014). Newly Documented Hybrids In The Tribe Boechereae (Brassicaceae) Challenge Current Generic Circumscriptions In The Group. Available online at: http://www.2014.botanyconference.org/engine/search/index.php? func= detail\&aid $=312$

Windham, M. D., Beck, J. B., Li, F.-W., Allphin, L., Carman, J. G., Sherwood, D. A., et al. (2015). Searching for diamonds in the apomictic rough: a case study involving Boechera lignifera (Brassicaceae). Syst. Bot. 40, 1031-1044. doi: $10.1600 / 036364415 \times 690076$

Conflict of Interest: The authors declare that the research was conducted in the absence of any commercial or financial relationships that could be construed as a potential conflict of interest.

Copyright (C) 2020 Mandáková, Hloušková, Windham, Mitchell-Olds, Ashby, Price, Carman and Lysak. This is an open-access article distributed under the terms of the Creative Commons Attribution License (CC BY). The use, distribution or reproduction in other forums is permitted, provided the original author(s) and the copyright owner(s) are credited and that the original publication in this journal is cited, in accordance with accepted academic practice. No use, distribution or reproduction is permitted which does not comply with these terms. 